\title{
AN IMPROVED TRAINING ALGORITHM IN HMM-BASED SPEECH RECOGNITION
}

\author{
Gongjun Li and Taiyi Huang \\ National Laboratory of Pattern Recognition \\ Chinese Academy of Sciences \\ Beijing 100080, China \\ \{ligj, huang\}@prldec3.ia.ac.cn
}

\begin{abstract}
In HMM-based speech recognition, estimation of parameters of HMMs is viewed as counterpart of training or learning in traditional sequential pattern recognition since speech signal can be represented by a sequence of $n$ dimension vectors after features are extracted from the speech signal. However, due to variation of duration of the phone with speakers and context and its randomness, speech samples contribute differently to estimation of parameters of HMMs. While only smaller training set is accessible, for instance, in the case of speaker adaptation, the problem becomes very serious.
\end{abstract}

In this paper, we analyze the impact of different duration of the phone on the output probability likelihood. To combat the above problem, two approaches are proposed to make proportionate the contribution of speech samples to estimation of parameters of HMM: geometrically averaged probability likelihood method and centralized parametric space method. Several experiments are conducted to verify the advantage of the above approaches in HMM-based speech recognition. The results show that the recognition rate can be improved to a certain degree when any one of the above approaches is employed.

\section{INTRODUCTION}

It is not a serious problem to estimate the parameters of HMMs including discrete HMM (DHMM), continuous HMM (CDHMM) and semi-continuous HMM (SCHMM) due to the famous Baum-Welch algorithm, in which the introduced Baum auxiliary function[1][2] makes separable the variables in ML-based objective function and growth transform accessible. Up to now HMM has been the mainstream in automatic speech recognition and much attention has been paid on HMM training, such as discriminative training[3][4], maximum mutual information based training[5] and minimum discriminative information based training[6].
In all these training algorithms, an assumption is implied that every speech sample contributes equally to estimation of parameters of HMMs and based upon this assumption, HMM-based speech recognition can be viewed as traditional sequential pattern recognition problem if speech signal is modeled as a sequence of $n$-dimension feature vectors. However, duration of the same phone varies with speakers and context, and in Chinese, also with tone pattern. On the other hand, the variation is of a little randomness. We found, in HMM-based speech recognition, that speech samples with different duration contribute differently to estimation of parameters of the same HMM and as a result, HMM models some speech units well and others bad. Consequently, the confusion among speech units is unavoidable. The fact that on the whole, duration of phones varies regularly with context and speakers, indicates that enlarging training set alone is not a perfect solution, although it is an available approach to the above problem because of somehow stochastic variation of duration of phones. Furthermore, while only smaller training set is accessible, for instance, in the case of speaker adaptation, the problem becomes very serious.

In this paper, we firstly analyze the impact of different duration of the phone on the output probability likelihood, in terms of which ML-based objective function is formed. To inhibit, even eliminate the effect of variation of duration, several approaches are presented to make proportionate the contribution of speech sample to HMM training, that is, geometrically averaged probability likelihood method and centralized parametric space method.

The organization of this paper is given as follows. At first we discuss the relation between duration of phones and the corresponding output probability likelihood in section 2. In next section two approaches are suggested to resolve the problem proposed as above. At last, based on Chinese allsyllable speech recognition task we conduct several experiments to verify the advantage of the above approaches over conventional MLE approach in HMMbased speech recognition. 


\section{DURATION OF PHONEMES AND OUTPUT PROBABILITY LIKELIHOOD}

If we denote speech sample w, the corresponding HMM pattern and output probability likelihood as $O_{T_{w}}^{w}, M_{w}$ and $P\left(O_{T_{w}}^{w} \mid M_{w}\right)$, respectively, and the training set consists of W speech samples, then ML-based objective function can be expressed as

$$
\prod_{w=1}^{W} P\left(O_{T_{w}}^{w} \mid M_{w}\right) \text { or } \sum_{w=1}^{W} \ln \left\{P\left(O_{T_{w}}^{w} \mid M_{w}\right)\right\}
$$

where $T_{w}$ is duration of speech sample w. Obviously, the output probability likelihood has a great influence on HMM training. Since the objective function is optimized over all speech samples, the comparative value of output probability likelihoods of all speech samples is the determinative factor.

If we denote the transition probability from state $\mathrm{i}$ to state $\mathrm{j}$ in $M_{w}$, output probability in which an observation $O_{t}^{w}$ is obtained in state $\mathrm{j}$, forward probability, backward probability and the corresponding output probability likelihood as $a_{i j}^{w}, b_{j}^{w}\left(O_{t}^{w}\right), \quad \alpha_{t}^{w}(i), \quad \beta_{t+1}^{w}(j)$ and $P_{w}$, respectively, then Forward-Backward recurrent formulas [1][2] for estimation of transition probability can given as follows

$$
\begin{gathered}
\gamma_{i j}^{w}=\frac{1}{P_{w}} \sum_{t=1}^{T_{w}} \alpha_{t}^{w}(i) a_{i j}^{w} b_{j}^{w}\left(O_{t+1}^{w}\right) \beta_{t+1}^{w}(j) \\
\gamma_{i}^{w}=\frac{1}{P_{w}} \sum_{t=1}^{T_{w}} \alpha_{t}^{w}(i) \beta_{t}^{w}(i) \\
\bar{a}_{i j}^{k}=\frac{\sum_{w: k \subset w} \gamma_{i j}^{w}}{\sum_{w: k \subset w} \gamma_{i}^{w}}
\end{gathered}
$$

As to speech samples of the same speech unit but of different duration, the expected number of transitions, $\gamma_{i j}^{w}$, from state $\mathrm{i}$ to state $\mathrm{j}$, and that of transitions, $\gamma_{i}{ }^{w}$, out of state $\mathrm{i}$, conditioned upon the observation sequence are closely relevant with their duration. Clearly, the duration of training samples determines their contribution to estimation of parameters of HMMs.

It is necessary to find out how the duration of speech samples influences the corresponding output probability likelihoods before we focus on how to combat the above problem. In HMM-based speech recognition larger duration of speech sample indicates more available paths or possible HMM state sequences. From the statistical data, we find that in some cases, the output probability likelihood increases with the duration of speech samples, but more often, speech samples of longer duration correspond to smaller output probability likelihoods. In HMM-based speech recognition Viterbi decoder is usually employed instead of computing output probability likelihood. A great number of experimental results show that no essential difference of recognition rate exists between the above both. It is rational to state that on the whole, the output probability along best path (that is, the output probability along this available path is the largest) can well represent the output probability likelihood for all speech samples. In speech recognition experiments we conduct, we find that the output probability along best path plays a prevailing role in HMM training over those along other available paths. In DHMM-based speech recognition, larger duration of speech samples corresponds to smaller output probability along best path since every transition probability and output probability in HMM is not greater than one. In CDHMMbased speech recognition, probability density can be much greater than one and the output probability along best path can increase with duration of speech samples.

If we neglect the impact of output probability along not-best path in objective function for HMM training, speech samples of longer duration contribute less to estimation of parameters of HMM in DHMM-based speech recognition. However, in CDHMM-based speech recognition longer duration influences HMM training inconsistently due to used probability density functions.

\section{TWO IMPROVED TRAINING APPROACHES}

It is explicit now that in traditional ML-based HMM training algorithm different duration of speech samples indicates different revision to the parameters of HMMs in Forward-Backward re-estimation formulas. Based on the above analysis we present two schemes to combat this problem, which are given as follows:

\subsection{Geometrically Averaged Output Probability Likelihood Method}

Comprehending the above analysis, we can make a conclusion that on the whole, the duration of speech samples influences the corresponding output probability likelihood without a certain regularity. If we quote the above definition, the objective function can be changed to be sum of geometrical average of output probability likelihood over all speech samples, that is

$$
\prod_{w=1}^{W}\left(P_{w}\right)^{1 / T_{w}} \text { or } \sum_{w=1}^{W} \ln \left(P_{w}^{1 / T_{w}}\right)
$$


Obviously, the function is not homogeneous polynomial with nonnegative coefficients any longer, and traditional Forward-Backward re-estimation formulas can not directly be used in this case. It is necessary that existence of a growth transform should be proved at first.

Theorem: Let $P=\left\{P_{w}\right\}$ be a set of homogeneous polynomials with nonnegative coefficients in its variables $\left\{x_{i}: x_{i}>0, \sum_{i} x_{i}=1\right\}$. As to such functions as $\prod_{w=1}^{W}\left(P_{w}\right)^{1 / T_{w}}$ the growth transform $x=\mathfrak{I}(x)$ exists, which guarantees the following equation correct

$$
\prod_{w=1}^{W}\left(P_{w}(\mathfrak{I}(x))\right)^{1 / T_{w}}>\prod_{w=1}^{W}\left(P_{w}(x)\right)^{1 / T_{w}}
$$

unless $\mathfrak{I}(x)=x$.

Proof.

According to stringent concavity of logarithmic function, we can obtain

$$
\begin{gathered}
\ln \left\{\frac{\prod_{w=1}^{W}\left(P_{w}\left(x^{i+1}\right)\right)^{1 / T_{w}}}{\prod_{w=1}^{W}\left(P_{w}\left(x^{i}\right)\right)^{1 / T_{w}}}\right\}=\sum_{w=1}^{W}\left\{\frac{1}{T_{w}} \cdot \ln \left(\frac{\left(P_{w}\left(x^{i+1}\right)\right)}{\left(P_{w}\left(x^{i}\right)\right)}\right)\right\} \\
\geq \sum_{w=1}^{W}\left\{\frac{1}{T_{w} \cdot P_{w}\left(x^{i}\right)} \cdot\left[\sum_{\theta}\left(P_{w}^{\theta}\left(x^{i}\right) \cdot\left(\ln \left(P_{w}^{\theta}\left(x^{i+1}\right)\right)-\ln \left(P_{w}^{\theta}\left(x^{i}\right)\right)\right)\right)\right]\right\} \mathrm{I}
\end{gathered}
$$

$\mathrm{n}$ the last expression variables are separated and the stringent concavity of logarithmic function guarantees that a globally optimal point objectively exists. Lemma 2 in [1] gives that a growth transform is accessible and make the following inequality correct

$$
\begin{aligned}
& \frac{1}{\sum_{w=1}^{W}\left(P_{w}\left(x^{i}\right)\right)^{1 / T_{w}}} \cdot \sum_{w=1}^{W}\left[\left(P_{w}\left(x^{i}\right)\right)^{1 / T_{w}} \cdot \ln \left(\frac{\left(P_{w}\left(x^{i+1}\right)\right)^{1 / T_{w}}}{\left(P_{w}\left(x^{i}\right)\right)^{1 / T_{w}}}\right)\right] \\
& \geq 0
\end{aligned}
$$

unless $x^{i+1}=x^{i}$.

Likewise, the above theorem is correct in the case of CDHMM and SCHMM.

Here we can obtain the re-estimation formulas for DHMM training as follows

$$
C_{w}=\frac{1}{T_{w} \cdot P_{w}}
$$

$$
\begin{gathered}
\gamma_{i j}^{w}=C_{w} \cdot \sum_{t=1}^{T_{w}} \alpha_{t}^{w}(i) a_{i j}^{w} b_{j}^{w}\left(O_{t+1}^{w}\right) \beta_{t+1}^{w}(j) \\
\gamma_{i}^{w}=C_{w} \cdot \sum_{t=1}^{T^{w}} \alpha_{t}^{w}(i) \beta_{t}^{w}(i) \\
\bar{a}_{i j}^{k}=\frac{\sum_{w: k \subset w} \gamma_{i j}^{w}}{\sum_{w: k \subset w} \gamma_{i}^{w}} \\
\bar{b}_{j k}^{i}=\frac{\sum_{w: i \subset w} \sum_{t \ni} O_{t}=v_{k}^{i}}{\sum_{w: i \subset w} \sum_{t=1}^{T^{w}} C_{t}^{w}(j) \beta_{t}^{w}(j)}
\end{gathered}
$$

Similar re-estimation formulas can derived to estimation the parameters of CDHMM and SCHMM.

\subsection{Centralized Parametric Space Method}

Observing the re-estimation formulas of transition probabilities of DHMM

$$
\bar{a}_{i j}^{k}=\frac{\sum_{w: k \subset w} \gamma_{i j}^{w}}{\sum_{w: k \subset w} \gamma_{i}^{w}}
$$

We can learn that $\gamma_{i}^{w}$ in the denominator can be strongly influenced by the duration of speech samples and therefore, if $\gamma_{i}^{m}$ of speech sample $\mathrm{m}$ is much greater than $\gamma_{i}^{n}$ of speech sample $\mathrm{n}$, the obtained HMM depends less on speech sample n. To make proportionate contribution of speech samples to estimation of parameters of HMMs, it is necessary to scale every component of the denominator in different rule, which relies on the duration of speech samples. For simplicity, we can select $\gamma_{i}^{n}$ as rule and it results in so-called centralized parametric space method. It can be realized as follows:

Step 1: During each iteration, compute the new parameters of HMMs based on every speech sample independently and keep them in memory separately.

Step 2: Revision of every speech sample to the same parameter is averaged over the number of all training samples or central points in parametric space of HMMs are treated as new parameters for next iteration.

Step 3: Go back to step 1 if final condition is not satisfied.

\section{EXPERIMENTAL RESULTS}


Here several experimental results are reported to verify the effectiveness of the proposed approaches. The experiments are conducted based on speaker-dependent Chinese allsyllable speech recognition. Speech representation is 12order LPC cepstrum coefficients. The initial and final are selected as recognition units. Three sets of utterances of 397 Chinese syllables are used for HMM training and other three sets form the test set. The experimental results are given in the following tables.

\section{Geometrically averaged output probability likelihood method}

This experiment is conducted based upon CDHMM-based speech recognition. The results are given in Table 1.

\begin{tabular}{|c|c|c|c|}
\hline $\begin{array}{c}\text { recognition } \\
\text { rate } \%\end{array}$ & $\begin{array}{c}\text { first- } \\
\text { candidate }\end{array}$ & $\begin{array}{c}\text { second- } \\
\text { candidate }\end{array}$ & $\begin{array}{c}\text { third- } \\
\text { candidate }\end{array}$ \\
\hline close-set & 92.6 & 97.7 & 99.2 \\
\hline open-set & 74.1 & 87.2 & 91.0 \\
\hline
\end{tabular}

Table 1.a Recognition rate in traditional CDHMM-based speech recognition

\begin{tabular}{|c|c|c|c|}
\hline $\begin{array}{c}\text { recognition } \\
\text { rate } \%\end{array}$ & $\begin{array}{c}\text { first- } \\
\text { candidate }\end{array}$ & $\begin{array}{c}\text { second- } \\
\text { candidate }\end{array}$ & $\begin{array}{c}\text { third- } \\
\text { candidate }\end{array}$ \\
\hline close-set & 93.1 & 98.4 & 99.1 \\
\hline open-set & 74.8 & 87.8 & 92.2 \\
\hline
\end{tabular}

Table 1.b Recognition rate with the improved CDHMM training algorithm

In the above experiments we find that geometrically averaging output probability likelihood can greatly inhibit the impact of duration of speech samples on their contribution to HMM training, but improve recognition rate slightly. We conduct another experiment in which two sets of utterances are used for HMM training and find out the effectiveness of the proposed training algorithm is finite, even trivial, if the training set is large enough. Another possible factor, which has negative effect on the improvement of recognition rate, is context-independent speech unit used in these experiments.

\section{Centralized parametric space method}

We conduct this experiment based on DHMM-based speech recognition task. The results are given as follows.

\begin{tabular}{|c|c|c|c|}
\hline $\begin{array}{c}\text { recognition } \\
\text { rate } \%\end{array}$ & $\begin{array}{c}\text { first- } \\
\text { candidate }\end{array}$ & $\begin{array}{c}\text { second- } \\
\text { candidate }\end{array}$ & $\begin{array}{c}\text { third- } \\
\text { candidate }\end{array}$ \\
\hline close-set & 86.3 & 96.7 & 99.1 \\
\hline open-set & 73.0 & 86.6 & 92.4 \\
\hline
\end{tabular}

Table 2.a Recognition rate in traditional DHMM-based speech recognition

\begin{tabular}{|c|c|c|c|}
\hline $\begin{array}{c}\text { recognition } \\
\text { rate } \%\end{array}$ & $\begin{array}{c}\text { first- } \\
\text { candidate }\end{array}$ & $\begin{array}{c}\text { second- } \\
\text { candidate }\end{array}$ & $\begin{array}{c}\text { third- } \\
\text { candidate }\end{array}$ \\
\hline close-set & 90.9 & 97.5 & 99.1 \\
\hline open-set & 78.2 & 91.2 & 95.3 \\
\hline
\end{tabular}

Table 2.b Recognition rate with the improved DHMM training algorithm

Clearly, centralized parametric space method can improve recognition rate remarkably though speech samples are enough. In contrast with geometrically averaged output probability likelihood method, scaling every component of the denominator in re-estimation formulas can more effectively make contribution of speech samples proportionate.

\section{CONCLUSION}

In this paper we present a problem in HMM training, that is, speech samples of different duration contribute differently to estimation of the parameters of HMMs. Based on profound analysis about it, two improved HMM training approaches are advanced. No major revision of ForwardBackward re-estimation formulas was required to the above schemes. Experimental results show that the proposed approaches can improve speech recognition to a certain degree.

\section{REFERENCES}

1. Levinson, S. and Rabiner, L. "An Introduction to the Application of the Theory of Probabilistic Functions of a Markov Process to Automatic Speech Recognition", B.S.T.J., Vol. 62(4), pp. 1035, 1983.

2. Juang, B.-H. and Labiner, L. "Mixture Autoregressive Hidden Markov Models for Speech Signals", IEEE trans. ASSP-33(6), pp. 1404, 1985.

3. Liu, C.-S., Lee, C.-H., and et al. "A Study on Minimum Error Discriminative Training for Speech Recognition", J.A.S.A., 97(1), pp. 637, 1995.

4. Juang, B.-H., and Katagiri, S. "Discriminative Learning for Minimum Error Classification”, IEEE trans. SP-40, pp. 3043, 1992.

5. Bahl, L., Brown, P., and et al. "Maximum Mutual Information Estimation of Hidden Markov Model Parameters for Speech Recognition", ICASSP'86, pp.49, 1986.

6. Ephraim, Y., Rabiner, L., and et al. "A Minimum Discrimination Information Approach for Hidden Markov Modeling", IEEE trans. IT-35(5), pp.1001, 1989. 